\title{
A SHORT NOTE ON REGULARITY CRITERIA FOR THE NAVIER-STOKES EQUATIONS CONTAINING THE VELOCITY GRADIENT
}

\author{
MILAN POKORNÝ \\ Mathematical Institute of Charles University \\ Sokolovská 83, 18675 Praha 8, Czech Republic \\ E-mail: pokorny@karlin.mff.cuni.cz
}

\begin{abstract}
We review several regularity criteria for the Navier-Stokes equations and prove some new ones, containing different components of the velocity gradient.
\end{abstract}

1. Introduction. We consider the Navier-Stokes equations

$$
\left.\begin{array}{c}
\frac{\partial \boldsymbol{u}}{\partial t}+\boldsymbol{u} \cdot \nabla \boldsymbol{u}-\Delta \boldsymbol{u}+\nabla p=\mathbf{0} \\
\operatorname{div} \boldsymbol{u}=0
\end{array}\right\} \text { in } I \times \Omega
$$

describing the flow of a viscous incompressible fluid. For simplicity, we put the constant density as well as the viscosity equal to 1 . We also take the right-hand side equal to zero. It is an easy matter to formulate all the criteria stated below for sufficiently smooth right-hand side.

To simplify the situation further, we have $\Omega=\mathbb{R}^{3}$. The same results can be obtained for $\Omega=(0, L)^{3}$ with the space-periodic boundary conditions. On the other hand, for $\Omega$ a smooth domain with homogeneous Dirichlet boundary conditions some proofs do not work. The main reason is a missing control on the pressure in the case of Dirichlet boundary conditions.

Finally, let the initial condition $\boldsymbol{u}_{0}$ be a sufficiently smooth $\left(\boldsymbol{u}_{0} \in W^{1,2}\left(\mathbb{R}^{3}\right)^{3}\right.$ at least $)$ divergence-free vector field.

Even though system (1) looks very innocently, in three space dimensions, the well-

2000 Mathematics Subject Classification: Primary 35Q30; Secondary 76D05.

Key words and phrases: Navier-Stokes equations; regularity of PDE's; uniqueness.

The work is a part of the research project MSM 0021620839 financed by MSMT and partly supported by the Grant Agency of the Charles University (grant No. 262/2002) and by the Czech Science Foundation (grant No. 201/02/P091).

The paper is in final form and no version of it will be published elsewhere. 
posedness in the sense of Hadamard is an open question since the work of Leray [13]. It is well known that for any $\boldsymbol{u}_{0} \in L^{2}\left(\mathbb{R}^{3}\right)^{3}$, $\operatorname{div} \boldsymbol{u}_{0}=0$ in $\mathcal{D}^{\prime}\left(\mathbb{R}^{3}\right)$ there exists at least one weak solution to (1) which satisfies the energy inequality

$$
\|\boldsymbol{u}(t)\|_{2}^{2}+2 \int_{0}^{t}\|\nabla \boldsymbol{u}\|_{2}^{2} d \tau \leq\left\|\boldsymbol{u}_{0}\right\|_{2}^{2} \quad \text { for all } t \in I
$$

and thus also $\lim _{t \rightarrow 0^{+}}\left\|\boldsymbol{u}(t)-\boldsymbol{u}_{0}\right\|_{2}=0$. Such solutions are called Leray-Hopf weak solutions.

On the other hand, the uniqueness and smoothness of the solution is known only either locally in time or for sufficiently small initial velocity. If $\Omega \subseteq \mathbb{R}^{2}$, the situation changes; we get both uniqueness of the weak solution and its regularity provided the data are smooth enough.

First regularity (and uniqueness) criteria were formulated already at the end of fifties and beginning of sixties; cf. [21], [24]. If

$$
\boldsymbol{u} \in L^{t}\left(I ; L^{s}\left(\mathbb{R}^{3}\right)^{3}\right), \quad \frac{2}{t}+\frac{3}{s} \leq 1, \quad s>3
$$

then the solution to (1) is smooth on $I$ and thus unique in the class of all Leray-Hopf weak solutions. Due to the scaling, the conditions $\frac{2}{t}+\frac{3}{s} \leq a$ will appear quite often. To simplify the notation, we will say that $\boldsymbol{u} \in(P S)_{a}$ if $\boldsymbol{u} \in L^{t}\left(I ; L^{s}\left(\mathbb{R}^{3}\right)\right)$ for some $s, t$ satisfying $\frac{2}{t}+\frac{3}{s}=a, s \in\left[\frac{3}{a}, \infty\right]$ and $\boldsymbol{u} \in \widetilde{(P S)_{a}}$ if $s \in\left(\frac{3}{a}, \infty\right]$ only. Thus the classical Prodi-Serrin condition can be formulated that $\boldsymbol{u} \in \widetilde{(P S)_{1}}$ implies regularity and uniqueness of the Leray-Hopf weak solutions.

This result was improved later on; first, it was shown (see [12]) that the case $\boldsymbol{u} \in$ $L^{\infty}\left(I ; L^{3}\left(\mathbb{R}^{3}\right)^{3}\right)$ implies uniqueness, later in a series of papers [8], [9] and [23] that this condition is also sufficient for smoothness, at least for $\Omega=\mathbb{R}^{3}$ and $\Omega=\mathbb{R}_{+}^{3}$.

Another improvement of the classical result can be found in [1]. The authors showed that for the regularity it is enough to have a certain information on two velocity components. The third component is estimated by the other two basically due to the divergencefree condition. A natural question appeared whether a certain regularity of one velocity component leads necessarily to the regularity of the whole vector field. This question was positively answered in the paper [16], where the authors showed that the needed regularity is

$$
u_{3} \in(P S)_{\frac{1}{2}} .
$$

(This result was proved as local regularity criterion for suitable weak solutions, however, it is an easy matter to transform it for the Cauchy problem.)

A very interesting combination of both criteria mentioned above can be found in [17]:

$$
\begin{aligned}
& u_{1}, u_{2} \in(P S)_{a}, 2 \leq t_{1} \leq \infty, 2 \leq s_{1} \leq \infty \\
& u_{3} \in(P S)_{b}, 2 \leq t_{2} \leq \infty, 3 \leq s_{2} \leq \infty \\
& a+b \leq 2,2 / t_{1}+2 / t_{2} \leq 1,2 / s_{1}+2 / s_{2}<1 .
\end{aligned}
$$

Further criteria can be formulated on the pressure $p$. Let us mention here only three such results. In [4] the authors showed that

$$
p \in \widetilde{(P S)_{2}}
$$


is enough to ensure the regularity. On the other hand, Seregin and Šverák showed that the boundedness of the pressure from below (i.e. without loss of generality, $p \geq 0$ ) is also sufficient. A local criterion for the non-negative part of the pressure was proved in [15]; however, both $p_{-} \in \widetilde{(P S)_{2}}$ and a certain regularity of the velocity in an arbitrarily small neighborhood of the point were needed.

A natural candidate for regularity criteria is the gradient of the velocity. One may guess that

$$
\nabla \boldsymbol{u} \in(P S)_{2}
$$

could yield the regularity. At least for $s<3$, it immediately follows from the classical Prodi-Serrin conditions and the results by Escauriaza, Seregin and Šverák due to the Sobolev imbedding theorem. Even though such a result has probably been known for a longer time, it was firstly published in [2] in 1995 (for $s>\frac{3}{2}$ ).

Evidently, if we replace $\nabla \boldsymbol{u}$ by the vorticity $\boldsymbol{\omega}=$ curl $\boldsymbol{u}$, we get the same criterion.

Similarly as for the velocity itself, there is a question whether one can formulate regularity criteria only for certain components of the velocity gradient or for certain components of the vorticity.

For the vorticity, it was shown in [5] that two components belonging to $\widetilde{(P S)_{2}}$ ensure the regularity. See also [17] for the discussion of the role of the vorticity. On the other hand, as was shown in [6] and further improved in [3], also certain smoothness of the vorticity direction guarantees the regularity.

The velocity gradient has nine components and the situation is much more complex. First result from [5] claimed that $\nabla u_{1}$ and $\nabla u_{2}$ belonging to $(P S)_{1}$ imply the regularity. This result is evidently not optimal in comparison to the vorticity. We will see that the condition $\widetilde{(P S)_{2}}$ is indeed sufficient, even for only two components of the velocity gradient. An interesting open question is whether the smoothness of only one vorticity component implies the regularity.

Next expected result,

$$
\nabla u_{3} \in(P S)_{\frac{3}{2}}
$$

guaranteeing the regularity was shown in [20]; independently also in [25]; particular case $s=3$ also in [24].

Further criteria will be mentioned in the following section. It was not our intention to mention all the criteria implying the regularity. One may replace the Lebesgue (Sobolev) spaces by the $B M O$ space, or even the Besov spaces, see e.g. [11] or [7]. Other interesting results can be found in [17], [18], where the regularity criteria are expressed via the eigenvalues or eigenfunctions of the symmetric part of the velocity gradient.

Note that, except for a certain logarithmic improvement of the Prodi-Serrin conditions (see [14]), the "optimal" condition for the velocity is $(P S)_{1}$ while for the pressure and the velocity gradient it is $(P S)_{2}$.

2. Regularity criteria containing components of the velocity gradient. We have already announced that $\nabla u_{3} \in(P S)_{\frac{3}{2}}$ guarantees the regularity. (In fact, we will slightly improve this result). It is not very surprising that $\frac{\partial \boldsymbol{u}}{\partial x_{3}} \in(P S)_{\frac{3}{2}}$ yields the same result. 
On the other hand, it might be more surprising that only two components, $\frac{\partial u_{3}}{\partial x_{3}}$ and $\frac{\partial u_{2}}{\partial x_{2}} \in \widetilde{(P S)_{2}}$ are also sufficient; but in this case, due to the divergence-free condition, we tacitly assume that the same regularity also holds for $\frac{\partial u_{1}}{\partial x_{1}}$. The result mentioned above implies the expected result that $\nabla u_{2}$ and $\nabla u_{3} \in \widetilde{(P S)_{2}}$ is sufficient for the regularity. It is possible to formulate also conditions for one component of the velocity gradient $\left(\frac{\partial u_{3}}{\partial x_{3}} \in L^{\infty}\left(I \times \mathbb{R}^{3}\right)\right.$, i.e. $\left.\frac{\partial u_{3}}{\partial x_{3}} \in(P S)_{0}\right)$. All the results mentioned above were proved in [19]. We will include all of them in Theorem 1 below, together with some new criteria whose sketch of the proof will be given in the last section.

THEOREM 1. Let $\boldsymbol{u}$ be a Leray-Hopf weak solution to the Navier-Stokes equations (1) corresponding to the initial velocity $\boldsymbol{u}_{0} \in W^{1,2}\left(\mathbb{R}^{3}\right)^{3}$, div $\boldsymbol{u}_{0}=0$. Let one of the following conditions be satisfied:

(a) three components
(i) $\frac{\partial u_{3}}{\partial x_{3}}, \frac{\partial u_{2}}{\partial x_{2}} \in \widetilde{(P S)_{2}}$ (then also $\frac{\partial u_{1}}{\partial x_{1}} \in \widetilde{(P S)_{2}}$ )
(ii) $\frac{\partial \boldsymbol{u}}{\partial x_{3}} \in(P S)_{\frac{3}{2}}$
(iii) $\frac{\partial u_{3}}{\partial x_{3}} \in(P S)_{1}, \frac{\partial u_{2}}{\partial x_{2}}, \frac{\partial u_{1}}{\partial x_{1}} \in \widetilde{(P S)_{2}}$
(iv) $\frac{\partial u_{3}}{\partial x_{3}} \in(P S)_{2}, 2 \leq s \leq 3, \frac{\partial u_{3}}{\partial x_{3}} \in(P S)_{\frac{5}{3}+\frac{1}{s}}, s>3, \frac{\partial u_{3}}{\partial x_{3}} \in(P S)_{\frac{11}{4}-\frac{3}{2 s}}, s \in\left[\frac{18}{11}, 2\right]$ and $\frac{\partial u_{3}}{\partial x_{1}}, \frac{\partial u_{3}}{\partial x_{2}} \in(P S)_{\frac{3}{2}}$
(v) $\frac{\partial u_{3}}{\partial x_{3}} \in(P S)_{2}, 2 \leq s \leq 3, \frac{\partial u_{3}}{\partial x_{3}} \in(P S)_{\frac{5}{3}+\frac{1}{s}}, s>3, \frac{\partial u_{3}}{\partial x_{3}} \in(P S)_{\frac{11}{4}-\frac{3}{2 s}}, s \in\left[\frac{18}{11}, 2\right]$ and $\frac{\partial u_{2}}{\partial x_{3}}, \frac{\partial u_{3}}{\partial x_{2}} \in(P S)_{\frac{3}{2}}$

(b) two components

(i) $\frac{\partial u_{1}}{\partial x_{3}}, \frac{\partial u_{2}}{\partial x_{3}} \in(P S)_{1}$
(ii) $\frac{\partial u_{2}}{\partial x_{3}} \in(P S)_{2}, s \in[2,3], \frac{\partial u_{2}}{\partial x_{3}} \in(P S)_{\frac{5}{3}+\frac{1}{s}}, s>3, \frac{\partial u_{2}}{\partial x_{3}} \in(P S)_{\frac{11}{4}-\frac{3}{2 s}}, s \in\left[\frac{18}{11}, 2\right]$ and $\frac{\partial u_{3}}{\partial x_{3}} \in(P S)_{1}$

(c) one component

(i) $\frac{\partial u_{3}}{\partial x_{3}} \in(P S)_{0}$.

Then $\boldsymbol{u}$ is a smooth solution to the Navier-Stokes equations, unique in the class of all Leray-Hopf weak solutions.

A very interesting problem are intermediate results between the special criteria. Unfortunately, the methods of the proof do not seem to cover these "interpolation" results. We leave this as a very interesting open problem.

3. Proof of new regularity criteria. We will sketch the proof of new criteria $(a)_{(v)}$ and $(b)_{(i)}-(b)_{(i i)}$. The proof of the remaining ones can be found in [19] or [20]. We only show a priori estimates for smooth solutions. The reader can find arguments how to deal with weak solutions e.g. in the papers mentioned a few lines above.

STEP 1: Proof of $(a)_{(v)}$. We will follow the proof of $(a)_{(i v)}$ given in [20]. There, the stronger condition $\frac{\partial u_{3}}{\partial x_{3}} \in(P S)_{\frac{3}{2}}$ was assumed. We will point out the argument how we improve the result. First, we have 
Lemma 1. Denote by $\omega_{3}$ the third component of the vorticity, $\omega_{3}=\frac{\partial u_{2}}{\partial x_{1}}-\frac{\partial u_{1}}{\partial x_{2}}$. Assume that $\frac{\partial u_{3}}{\partial x_{3}} \in \widetilde{(P S)_{2}}$ and $\frac{\partial u_{3}}{\partial x_{2}}, \frac{\partial u_{2}}{\partial x_{3}} \in(P S)_{\frac{3}{2}}$. Then there exist $C_{1}$ and $C_{2}$ depending only on the initial condition and the norms mentioned above such that

$$
\left\|\omega_{3}\right\|_{L^{\infty}\left(I ; L^{2}\left(\mathbb{R}^{3}\right)\right) \cap L^{2}\left(I ; L^{6}\left(\mathbb{R}^{3}\right)\right)}^{2} \leq C_{1}+C_{2}\|\boldsymbol{\omega}\|_{L^{\infty}\left(I ; L^{2}\left(\mathbb{R}^{3}\right)\right) \cap L^{2}\left(I ; L^{6}\left(\mathbb{R}^{3}\right)\right)} .
$$

REMARK 1. Lemma 1 holds if we replace $\frac{\partial u_{2}}{\partial x_{3}}$ by $\frac{\partial u_{3}}{\partial x_{1}}$.

Proof. We have

$$
\begin{gathered}
\frac{1}{2} \frac{d}{d t}\left\|\omega_{3}\right\|_{2}^{2}+\left\|\nabla \omega_{3}\right\|_{2}^{2}=\int_{\mathbb{R}^{3}}(\boldsymbol{\omega} \cdot \nabla) u_{3} \omega_{3} \\
=\int_{\mathbb{R}^{3}} \omega_{3}^{2} \frac{\partial u_{3}}{\partial x_{3}}+\int_{\mathbb{R}^{3}} \omega_{2} \omega_{3} \frac{\partial u_{3}}{\partial x_{2}}+\int_{\mathbb{R}^{3}}\left(\frac{\partial u_{3}}{\partial x_{2}}-\frac{\partial u_{2}}{\partial x_{3}}\right) \omega_{3} \frac{\partial u_{3}}{\partial x_{1}} .
\end{gathered}
$$

The Hölder inequality applied in a different way on the first, and on the last two terms together with the Gronwall inequality (see the estimates in [20]) yield the result.

Next, consider the momentum equation written in the form

$$
\frac{\partial \boldsymbol{u}}{\partial t}-\Delta \boldsymbol{u}+(\boldsymbol{\omega} \times \boldsymbol{u})+\nabla\left(p+\frac{1}{2}|\boldsymbol{u}|^{2}\right)=\mathbf{0},
$$

multiply it by $\Delta \boldsymbol{u}$ and integrate over $\mathbb{R}^{3}$. Using the Green theorem the equality yields

$$
\frac{1}{2} \frac{d}{d t}\|\nabla \boldsymbol{u}\|_{2}^{2}+\left\|\nabla^{2} \boldsymbol{u}\right\|_{2}^{2}=\int_{\mathbb{R}^{3}}(\boldsymbol{\omega} \times \boldsymbol{u}) \cdot \Delta \boldsymbol{u} .
$$

Recall that

$$
(\boldsymbol{\omega} \times \boldsymbol{u}) \cdot \Delta \boldsymbol{u}=\left(\omega_{2} u_{3}-\omega_{3} u_{2}\right) \Delta u_{1}+\left(\omega_{3} u_{1}-\omega_{1} u_{3}\right) \Delta u_{2}+\left(\omega_{1} u_{2}-\omega_{2} u_{1}\right) \Delta u_{3} .
$$

Terms containing $\omega_{1}$ and $\omega_{3}$ can be estimated as $I_{22}$ and $I_{23}$ in [20]. Thus we have to control

$$
\begin{gathered}
\int_{\mathbb{R}^{3}}\left(\omega_{2} u_{3} \Delta u_{1}-\omega_{2} u_{1} \Delta u_{3}\right)=\int_{\mathbb{R}^{3}}\left(\frac{\partial \omega_{2}}{\partial x_{k}} u_{1} \frac{\partial u_{3}}{\partial x_{k}}-\frac{\partial \omega_{2}}{\partial x_{k}} u_{3} \frac{\partial u_{1}}{\partial x_{k}}\right) \\
=-\int_{\mathbb{R}^{3}} \frac{\partial^{2} u_{1}}{\partial x_{3} \partial x_{k}} u_{3} \frac{\partial u_{1}}{\partial x_{k}}+\int_{\mathbb{R}^{3}} \frac{\partial^{2} u_{1}}{\partial x_{3} \partial x_{k}} u_{1} \frac{\partial u_{3}}{\partial x_{k}}+\int_{\mathbb{R}^{3}} \frac{\partial^{2} u_{3}}{\partial x_{1} \partial x_{k}} u_{3} \frac{\partial u_{1}}{\partial x_{k}}-\int_{\mathbb{R}^{3}} \frac{\partial^{2} u_{3}}{\partial x_{1} \partial x_{k}} u_{1} \frac{\partial u_{3}}{\partial x_{k}} .
\end{gathered}
$$

The first term, $\frac{1}{2} \int_{\mathbb{R}^{3}} \frac{\partial u_{1}}{\partial x_{k}} \frac{\partial u_{1}}{\partial x_{k}} \frac{\partial u_{3}}{\partial x_{3}}$, can be estimated as $I_{21}$ in [20] for $\frac{\partial u_{3}}{\partial x_{3}} \in \widetilde{(P S)_{2}}$. The second term contains for $k=2 \frac{\partial u_{3}}{\partial x_{2}}$ and for $k=3 \frac{\partial u_{3}}{\partial x_{3}}$-here we need the stronger assumptions for $s<2$ and $s>3$, see the estimates below. If $k=1$, we use the continuity equation and

$$
\begin{gathered}
\int_{\mathbb{R}^{3}} \frac{\partial^{2} u_{1}}{\partial x_{3} \partial x_{1}} u_{1} \frac{\partial u_{3}}{\partial x_{1}}=-\int_{\mathbb{R}^{3}} \frac{\partial^{2} u_{2}}{\partial x_{3} \partial x_{2}} u_{1} \frac{\partial u_{3}}{\partial x_{1}}-\int_{\mathbb{R}^{3}} \frac{\partial^{2} u_{3}}{\partial^{2} x_{3}} u_{1} \frac{\partial u_{3}}{\partial x_{1}} \\
=\int_{\mathbb{R}^{3}} \frac{\partial u_{2}}{\partial x_{3}}\left(\frac{\partial u_{1}}{\partial x_{2}} \frac{\partial u_{3}}{\partial x_{1}}+u_{1} \frac{\partial^{2} u_{3}}{\partial x_{2} \partial x_{1}}\right)+\int_{\mathbb{R}^{3}} \frac{\partial u_{3}}{\partial x_{3}} \frac{\partial u_{1}}{\partial x_{3}} \frac{\partial u_{3}}{\partial x_{1}}-\frac{1}{2} \int_{\mathbb{R}^{3}} \frac{\partial u_{3}}{\partial x_{3}} \frac{\partial u_{3}}{\partial x_{3}} \frac{\partial u_{1}}{\partial x_{1}} .
\end{gathered}
$$


Similarly can also be treated the other terms. Now, if $s<2$,

$$
\begin{aligned}
\left|\int_{\mathbb{R}^{3}} \frac{\partial u_{3}}{\partial x_{3}} u_{i} \frac{\partial^{2} u_{j}}{\partial x_{k} \partial x_{l}}\right| & \leq C\left\|\frac{\partial u_{3}}{\partial x_{3}}\right\|\|\nabla \boldsymbol{u}\|_{2}^{\frac{1}{2}}\left\|\nabla^{2} \boldsymbol{u}\right\|_{2}^{\frac{3}{2}} \leq C\left\|\frac{\partial u_{3}}{\partial x_{3}}\right\|_{s}^{\frac{2 s}{6-s}}\|\nabla \boldsymbol{u}\|_{2}^{\frac{1}{2}}\left\|\nabla^{2} \boldsymbol{u}\right\|_{2}^{\frac{30-9 s}{2(6-s)}} \\
& \leq \frac{1}{2}\left\|\nabla^{2} \boldsymbol{u}\right\|_{2}^{2}+C\left\|\frac{\partial u_{3}}{\partial x_{3}}\right\|_{s}^{\frac{8 s}{5 s-6}}\|\nabla \boldsymbol{u}\|_{2}^{2+\frac{24-12 s}{5 s-6}} \\
\leq & \frac{1}{2}\left\|\nabla^{2} \boldsymbol{u}\right\|_{2}^{2}+C\|\nabla \boldsymbol{u}\|_{2}^{2}\left(\left\|\frac{\partial u_{3}}{\partial x_{3}}\right\|_{s}^{\frac{8 s}{11 s-18}}+\|\nabla \boldsymbol{u}\|_{2}^{2}\right) .
\end{aligned}
$$

If $s>3$,

$$
\begin{gathered}
\left|\int_{\mathbb{R}^{3}} \frac{\partial u_{3}}{\partial x_{3}} u_{i} \frac{\partial^{2} u_{j}}{\partial x_{k} \partial x_{l}}\right| \leq\left\|\frac{\partial u_{3}}{\partial x_{3}}\right\|_{s}^{\frac{3 s}{5 s-6}}\|\nabla \boldsymbol{u}\|_{2}^{2 \frac{s-3}{5 s-6}}\left\|\nabla^{2} \boldsymbol{u}\right\|_{2}\|\boldsymbol{u}\|_{\frac{2}{3} \frac{5 s-6}{s-2}} \\
\leq C\left\|\frac{\partial u_{3}}{\partial x_{3}}\right\|_{s}^{\frac{3 s}{5 s-6}}\|\nabla \boldsymbol{u}\|_{2}\left\|\nabla^{2} \boldsymbol{u}\right\|_{2}\|\boldsymbol{u}\|_{2}^{2 \frac{s-3}{5 s-6}} \leq \frac{1}{2}\left\|\nabla^{2} \boldsymbol{u}\right\|_{2}^{2}+C\left\|\frac{\partial u_{3}}{\partial x_{3}}\right\|_{s}^{\frac{6 s}{5 s-6}}\|\nabla \boldsymbol{u}\|_{2}^{2} .
\end{gathered}
$$

These two estimates correspond exactly to conditions $(a)_{(i v)}$ and $(a)_{(v)}$ for $\frac{\partial u_{3}}{\partial x_{3}}$.

STEP 2: Proof of $(b)_{(i)}$. The proof is relatively simple. We will show that $(b)_{(i)}$ implies $\frac{\partial \boldsymbol{u}}{\partial x_{3}} \in L^{\infty}\left(I ; L^{2}\left(\mathbb{R}^{3}\right)^{3}\right)$, i.e. $\frac{\partial \boldsymbol{u}}{\partial x_{3}} \in(P S)_{\frac{3}{2}}$. We have

$$
\frac{1}{2} \frac{d}{d t}\left\|\frac{\partial \boldsymbol{u}}{\partial x_{3}}\right\|_{2}^{2}+\left\|\nabla \frac{\partial \boldsymbol{u}}{\partial x_{3}}\right\|_{2}^{2}=-\int_{\mathbb{R}^{3}} \frac{\partial u_{i}}{\partial x_{3}} \frac{\partial u_{j}}{\partial x_{3}} \frac{\partial u_{i}}{\partial x_{j}} .
$$

The only term which does not contain $\frac{\partial u_{i}}{\partial x_{3}}, i=1,2$ is

$$
\begin{aligned}
& -\int_{\mathbb{R}^{3}}\left(\frac{\partial u_{3}}{\partial x_{3}}\right)^{3}=2 \int_{\mathbb{R}^{3}} u_{3} \frac{\partial u_{3}}{\partial x_{3}} \frac{\partial^{2} u_{3}}{\partial x_{3}^{2}}=-2 \int_{\mathbb{R}^{3}} u_{3} \frac{\partial u_{3}}{\partial x_{3}}\left(\frac{\partial^{2} u_{1}}{\partial x_{1} \partial x_{3}}+\frac{\partial^{2} u_{2}}{\partial x_{2} \partial x_{3}}\right) \\
& =2 \int_{\mathbb{R}^{3}}\left(\frac{\partial u_{3}}{\partial x_{1}} \frac{\partial u_{3}}{\partial x_{3}}+u_{3} \frac{\partial^{2} u_{3}}{\partial x_{1} \partial x_{3}}\right) \frac{\partial u_{1}}{\partial x_{3}}+2 \int_{\mathbb{R}^{3}}\left(\frac{\partial u_{3}}{\partial x_{2}} \frac{\partial u_{3}}{\partial x_{3}}+u_{3} \frac{\partial^{2} u_{3}}{\partial x_{2} \partial x_{3}}\right) \frac{\partial u_{2}}{\partial x_{3}} .
\end{aligned}
$$

The first and the third term can be estimated by

$$
\left\|\frac{\partial u_{i}}{\partial x_{3}}\right\|_{s}\left\|\frac{\partial u_{3}}{\partial x_{3}}\right\|_{\frac{2 s}{s-2}}\|\nabla \boldsymbol{u}\|_{2}
$$

while the second and the fourth one by

$$
\left\|\frac{\partial u_{i}}{\partial x_{3}}\right\|_{s}\|\boldsymbol{u}\|_{\frac{2 s}{s-2}}\left\|\nabla \frac{\partial \boldsymbol{u}}{\partial x_{3}}\right\|_{2}
$$

Altogether

$$
\begin{gathered}
\left|\int_{\mathbb{R}^{3}} \frac{\partial u_{i}}{\partial x_{3}} \frac{\partial u_{j}}{\partial x_{3}} \frac{\partial u_{i}}{\partial x_{j}}\right| \leq \frac{1}{2}\left\|\nabla \frac{\partial \boldsymbol{u}}{\partial x_{3}}\right\|_{2}^{2} \\
+C\left(\|\boldsymbol{u}\|_{L^{\infty}\left(I ; L^{2}\left(\mathbb{R}^{3}\right)^{3}\right)}\right)\left(\left\|\frac{\partial u_{3}}{\partial x_{3}}\right\|_{s}^{\frac{2 s}{s-3}}+\|\nabla \boldsymbol{u}\|_{2}^{2}\right)\left(1+\left\|\frac{\partial u_{3}}{\partial x_{3}}\right\|_{2}^{2 \frac{s-3}{2 s-3}}\right)
\end{gathered}
$$

which implies the result. 
STEP 3: Proof of $(b)_{(i i)}$. One important tool is a modification of Theorem 1 from [19]. We have

Lemma 2. Let $\boldsymbol{u}$ be a Leray-Hopf weak solution to the Navier-Stokes equations (1) corresponding to the initial velocity $\boldsymbol{u}_{0} \in W^{1,2}\left(\mathbb{R}^{3}\right)^{3}$, $\operatorname{div} \boldsymbol{u}_{0}=0$. Let $u_{3} \in \widetilde{(P S)_{1}}$ and $\frac{\partial u_{2}}{\partial x_{3}}$, $\frac{\partial u_{3}}{\partial x_{3}}$ belong to $(P S)_{2}, s \in[2,3]$, to $(P S)_{\frac{5}{3}+\frac{1}{s}}, s>3$ and to $(P S)_{\frac{11}{4}-\frac{3}{2 s}}, s \in\left[\frac{18}{11}, 2\right]$. Then $\boldsymbol{u}$ is a smooth solution to the Navier-Stokes equations, unique in the class of all Leray-Hopf weak solutions.

Proof. We proceed as in the proof of Theorem 1 mentioned above. We have

$$
\frac{1}{2} \frac{d}{d t}\|\boldsymbol{\omega}\|_{2}^{2}+\|\nabla \boldsymbol{\omega}\|_{2}^{2}=\int_{\mathbb{R}^{3}} \omega_{i} \frac{\partial u_{i}}{\partial x_{j}} \omega_{j} .
$$

The right-hand side can be written as

$$
\begin{gathered}
\int_{\mathbb{R}^{3}} \frac{\partial u_{2}}{\partial x_{3}} \frac{\partial u_{2}}{\partial x_{3}} \frac{\partial u_{1}}{\partial x_{1}}-\int_{\mathbb{R}^{3}} \frac{\partial u_{2}}{\partial x_{1}} \frac{\partial u_{2}}{\partial x_{3}} \frac{\partial u_{1}}{\partial x_{3}}+\int_{\mathbb{R}^{3}} \frac{\partial u_{1}}{\partial x_{3}} \frac{\partial u_{1}}{\partial x_{3}} \frac{\partial u_{2}}{\partial x_{2}} \\
-\int_{\mathbb{R}^{3}} \frac{\partial u_{1}}{\partial x_{2}} \frac{\partial u_{2}}{\partial x_{3}} \frac{\partial u_{1}}{\partial x_{3}}+\int_{\mathbb{R}^{3}} c_{i j k l m} u_{3} \frac{\partial u_{i}}{\partial x_{j}} \frac{\partial^{2} u_{k}}{\partial x_{l} \partial x_{m}} .
\end{gathered}
$$

The only term which cannot be handled as in the article mentioned above is

$$
\int_{\mathbb{R}^{3}} \frac{\partial u_{1}}{\partial x_{3}} \frac{\partial u_{1}}{\partial x_{3}} \frac{\partial u_{2}}{\partial x_{2}}=-\int_{\mathbb{R}^{3}} \frac{\partial u_{1}}{\partial x_{3}} \frac{\partial u_{1}}{\partial x_{3}} \frac{\partial u_{1}}{\partial x_{1}}-\int_{\mathbb{R}^{3}} \frac{\partial u_{1}}{\partial x_{3}} \frac{\partial u_{1}}{\partial x_{3}} \frac{\partial u_{3}}{\partial x_{3}} .
$$

The last term can be easily estimated. We apply the Green theorem in the first term on the right-hand side and use once more the continuity equation. Then

$$
\begin{gathered}
-\int_{\mathbb{R}^{3}} \frac{\partial u_{1}}{\partial x_{3}} \frac{\partial u_{1}}{\partial x_{3}} \frac{\partial u_{1}}{\partial x_{1}}=-2 \int_{\mathbb{R}^{3}} \frac{\partial}{\partial x_{3}}\left(\frac{\partial u_{2}}{\partial x_{2}}+\frac{\partial u_{3}}{\partial x_{3}}\right) u_{1} \frac{\partial u_{1}}{\partial x_{3}} \\
=2 \int_{\mathbb{R}^{3}} \frac{\partial u_{2}}{\partial x_{3}}\left(u_{1} \frac{\partial^{2} u_{1}}{\partial x_{2} \partial x_{3}}+\frac{\partial u_{1}}{\partial x_{2}} \frac{\partial u_{1}}{\partial x_{3}}\right)+2 \int_{\mathbb{R}^{3}} \frac{\partial u_{3}}{\partial x_{3}}\left(u_{1} \frac{\partial^{2} u_{1}}{\partial x_{3}^{2}}+\frac{\partial u_{1}}{\partial x_{3}} \frac{\partial u_{1}}{\partial x_{3}}\right) .
\end{gathered}
$$

We have

$$
\left|\int_{\mathbb{R}^{3}} \frac{\partial u_{i}}{\partial x_{3}} u_{1} \frac{\partial^{2} u_{1}}{\partial x_{j} \partial x_{k}}\right| \leq\left\|\frac{\partial u_{i}}{\partial x_{3}}\right\|_{s}\|\boldsymbol{u}\|_{\frac{2 s}{s-2}}\left\|\nabla^{2} \boldsymbol{u}\right\|_{2} \leq \frac{1}{2}\|\nabla \boldsymbol{\omega}\|_{2}^{2}+C\|\boldsymbol{\omega}\|_{2}^{2}\left\|\frac{\partial u_{2}}{\partial x_{3}}\right\|_{s}^{\frac{2 s}{2 s-3}},
$$

provided $s \in[2,3]$. For $s>3$ or $s<2$ we may use the estimates at the end of Step 1 .

Now, we proceed as in the proof of $(a)_{(i i i)}$ in [19]. Assume that $\frac{\partial u_{2}}{\partial x_{3}}$ satisfy $(b)_{(i i)}$ and $\frac{\partial u_{3}}{\partial x_{3}} \in(P S)_{1}$. Then, as $\frac{\partial u_{3}}{\partial x_{3}} \in L^{2}\left(I \times \mathbb{R}^{3}\right)$, it is an easy matter to see that $\frac{\partial u_{3}}{\partial x_{3}} \in$ $L^{2}\left(I ; L^{3}\left(\mathbb{R}^{3}\right)\right)$ and thus it satisfies the assumption of Lemma 2 . It remains to estimate $u_{3}$ in $\widetilde{(P S)_{1}}$. We have

$$
\frac{1}{3} \frac{d}{d t}\left\|u_{3}\right\|_{3}^{3}+\frac{8}{9}\left\|\nabla\left|u_{3}\right|^{\frac{3}{2}}\right\|_{2}^{2}=-\int_{\mathbb{R}^{3}} \frac{\partial p}{\partial x_{3}}\left|u_{3}\right| u_{3} .
$$

We apply the Green theorem in the term on the right-hand side and, using standard estimates of the pressure, we get

$$
\left|\int_{\mathbb{R}^{3}} \frac{\partial p}{\partial x_{3}}\right| u_{3}\left|u_{3}\right| \leq C\left(\left\|\frac{\partial u_{3}}{\partial x_{3}}\right\|_{s}^{\frac{2 s}{s-3}}+\|\boldsymbol{u}\|_{6}^{2}\right)\|\boldsymbol{u}\|_{2}^{\frac{s-3}{s}}\left\|u_{3}\right\|_{3},
$$

i.e. $u_{3} \in \widetilde{(P S)_{1}}$ provided $\frac{\partial u_{3}}{\partial x_{3}} \in(P S)_{1}$. The proof of $(b)_{(i i)}$ is complete. 


\section{References}

[1] H. O. Bae and H. J. Choe, $L^{\infty}$-bound of weak solutions to Navier-Stokes equations, in: Proceedings of Korea-Japan Partial Differential Equations Conference (Taejon, 1996), H. J. Choe et al. (eds.), Seoul National University, 1997, 13 pp.

[2] H. Beirão da Veiga, A new regularity class for the Navier-Stokes equations in $R^{n}$, Chin. Ann. Math. Ser. B 16 (1995), 407-412.

[3] H. Beirão da Veiga and L. Berselli, On the regularizing effect of the vorticity direction in incompressible viscous flows, Differential Integral Equations 15 (2002), 345-356.

[4] L. Berselli and P. G. Galdi, Regularity criteria involving the pressure for the weak solutions to the Navier-Stokes equations, Proc. Am. Math. Soc. 130 (2002), 3585-3595.

[5] D. Chae and H.J. Choe, Regularity of solutions to the Navier-Stokes equation, Electron. J. Differ. Equ. 5 (1999), 1-7.

[6] P. Constantin and C. Fefferman, Direction of vorticity and the problem of global regularity for the Navier-Stokes equations, Indiana Univ. Math. J. 42 (1993), 775-789.

[7] R. Danchin, Zero Mach number limit in critical spaces for compressible Navier-Stokes equations, Ann. Sci. Ec. Norm. Sup. IV 35 (2002), 27-75.

[8] L. Escauriaza, G. Seregin and V. Šverák, Backward uniqueness for parabolic equations, Arch. Ration. Mech. Anal. 169 (2003), 147-157.

[9] L. Escauriaza, G. Seregin and V. Šverák, Backward uniqueness for the heat operator in a half-space, St. Petersbg. Math. J. 15 (2004), 139-148.

[10] C. He, Regularity for solutions to the Navier-Stokes equations with one velocity component regular, Electron. J. Differential Equations 29 (2002) 1-13.

[11] H. Kozono and Y. Taniuchi, Bilinear estimates in BMO and the Navier-Stokes equations, Math. Z. 235 (2000), 173-194.

[12] H. Kozono and H. Sohr, Remark on uniqueness of weak solutions to the Navier-Stokes equations, Analysis 16 (1996), 255-271.

[13] J. Leray, Sur le mouvement d'un liquide visqueux emplissant l'espace, Acta Math. 63 (1934), 193-248.

[14] S. Montgomery-Smith, Conditions implying regularity of the three dimensional NavierStokes equations, to appear in Appl. Math.

[15] J. Neustupa and J. Nečas, New conditions for local regularity of a suitable weak solution to the Navier-Stokes equations, J. Math. Fluid Mech. 4 (2002), 237-256.

[16] J. Neustupa, A. Novotný and P. Penel, An interior regularity of a weak solution to the Navier-Stokes equations in dependence on one component of velocity, in: Topics in Mathematical Fluid Mechanics, Quad. Mat. 10 (2002), 163-183; see also A remark to interior regularity of a suitable weak solution to the Navier-Stokes equations, CIM preprint No. 25 (1999).

[17] J. Neustupa and P. Penel, Anisotropic and geometric criteria for interior regularity of weak solutions to the $3 D$ Navier-Stokes Equations, in: Mathematical Fluid Mechanics (Recent Results and Open Problems), J. Neustupa and P. Penel (eds.), Advances in Mathematical Fluid Mechanics, Birkhäuser, 2001, 239-267.

[18] J. Neustupa and P. Penel, The role of eigenvalues and eigenvectors of the symmetrized gradient of velocity in the theory of the Navier-Stokes equations, C. R. Math. Acad. Sci. Paris 336 (2003), 805-810.

[19] P. Penel and M. Pokorný, Some new regularity criteria for the Navier-Stokes equations containing the gradient of velocity, Appl. Math. 49 (2004), 483-493; see also preprint of Charles University MATH-MU-9/2001. 
[20] M. Pokorný, On the result of He concerning the smoothness of solutions to the NavierStokes equations, Electron. J. Differ. Equ. 11 (2003), 1-8.

[21] G. Prodi, Un teorema di unicità per el equazioni di Navier-Stokes, Ann. Mat. Pura Appl. IV 48 (1959), 173-182.

[22] G. Seregin and V. Šverák, Navier-Stokes with lower bounds on the pressure, Arch. Ration. Mech. Anal. 163 (2002), 65-86.

[23] G. Seregin and V. Šverák, The Navier-Stokes equations and backward uniqueness, in: Nonlinear Problems in Mathematical Physics and Related Topics II. In honour of Professor O. A. Ladyzhenskaya, M. Sh. Birman et al. (eds.), Kluwer, 2002, 353-366.

bibitemSe J. Serrin, The initial value problems for the Navier-Stokes equations, in: Nonlinear Problems, R. E. Langer (ed.), University of Wisconsin Press, 1963.

[24] Z. Skalák and P. Kučera, A note on coupling of velocity components for the Navier-Stokes equations, Z. Angew. Math. Mech. 84 (2004), 124-127.

[25] Y. Zhou, A new regularity result for the Navier-Stokes equations in terms of the gradient of one velocity component, Methods Appl. Anal. 9 (2002), 563-578. 